The Journal of Nonlinear Siences and $_{\text {Applications }}$

http://www.tjnsa.com

\title{
CONVERGENCE THEOREMS FOR THE ZEROS OF A FINITE FAMILY OF GENERALIZED $\Phi$-ACCRETIVE OPERATORS
}

\author{
N. GURUDWAN ${ }^{1}$, B. K. SHARMA ${ }^{2}$
}

\begin{abstract}
A strong convergence theorem for the common zero for a finite family of Generalized Lipschitz operators in a uniformly smooth Banach space is proved when atleast one of the operator is Generalized $\Phi$ - accretive, using a new iteration formula. Similar result for Generalized Lipschitz and Generalized $\Phi$ - pseudocontractive map is also proved. Our result extends the convergence results of Chidume 4 to a finite family improving many other results.
\end{abstract}

\section{Introduction AND Preliminaries}

Hirano [5] studied the Mann iteration process introduced by $\mathrm{Xu}$ [11],

$$
x_{n+1}=a_{n} x_{n}+b_{n} v_{n}+c_{n} u_{n}, \forall v_{n} \in S x_{n}, n \geq 0,
$$

to prove the strong convergence of a multi-valued $\phi$-strongly accretive operator with a bounded range in a uniformly smooth Banach space setting. This result itself is a generalization of many of the previous results (see [5] and the references therein).

Recently, Chidume and Chidume [4] extended the work to a more general class of Generalized Lipschitz and Generalized $\Phi$-quasi-accretive operator in the same space setting.

Within the past 10 years or so, considerable research efforts have been devoted to developing iterative methods for approximating common fixed points (assuming existence) for families of several classes of nonlinear mappings et al. [3, 6, 10, 13]. Markov [8] showed that if a commutating family of bounded linear transformations $T_{\alpha}, \alpha \in \Delta$, ( $\Delta$ an arbitrary index set) of a normed linear space $\mathrm{E}$ into itself leaves some nonempty compact convex subset $\mathrm{K}$ of E invariant, then the family has at least one common fixed point. Motivated by this result, De Marr [7] studied the problem of the existence of a common fixed point for a family of

2000 Mathematics Subject Classification. 47H06, 47H09.

Key words and phrases. Generalized $\Phi$-accretive; generalized Lipschitz; uniformly smooth Banach space; mann iteration. 
nonlinear maps.

In this paper, we introduce a new iteration process and prove that it converges strongly to a common zero for a finite family of generalized Lipschitz nonlinear mappings in a uniformly smooth Banach space if at least one member of the family is Generalized $\Phi$-accretive. We also prove that a slight modification of the process converges to a common fixed point for a finite family of generalized Lipschitz pseudocontractive operators defined on E. Thus our result extends [4] to a finite family of generalized Lipschitz and generalized $\Phi$-accretive operator, which itself is a generalization of many of the previous results.

Let $E$ be a real Banach space with dual $E^{*}$. The normalized duality mapping from $E$ to $2^{E^{*}}$ is defined by

$$
J(x):=\left\{x^{*} \in E^{*}:\left\langle x, x^{*}\right\rangle=\|x\|^{2},\|x\|=\left\|x^{*}\right\|\right\},
$$

where $\langle.,$.$\rangle denotes the duality pairing between the elements of E$ and $E^{*}$.

Definition 1.1. ([1]) A mapping $A: D(A)=E \rightarrow E$ is said to be accretive if for all $x, y \in E$, there exists $j(x-y) \in J(x-y)$ such that

$$
\langle A x-A y, j(x-y)\rangle \geq 0 .
$$

The mapping $A$ is said to be $\phi$ - strongly accretive if there is a strictly increasing function $\phi:[0, \infty) \rightarrow[0, \infty)$ with $\phi(0)=0$ such that for any $x, y \in E$, there exists $j(x-y) \in J(x-y)$ such that

$$
\langle A x-A y, j(x-y)\rangle \geq \phi(\|x-y\|)\|x-y\|
$$

and is said to be Generalized $\Phi$-accretive [4] if there is a strictly increasing function $\Phi$ : $[0, \infty) \rightarrow[0, \infty)$ with $\Phi(0)=0$ such that for any $x, y \in E$, there exists $j(x-y) \in J(x-y)$ such that

$$
\langle A x-A y, j(x-y)\rangle \geq \Phi(\|x-y\|) .
$$

It is well known [4] that the class of generalized $\Phi$-accretive mappings includes the class of $\phi$-accretive strongly accretive operators as a special case (put $\Phi(s)=s \phi(s)$ for all $s \in[0, \infty)$ ).

Definition 1.2. ([2]) The mapping $T: E \rightarrow E$ is called pseudocontractive if for all $x, y \in E$, there exists $j(x-y) \in J(x-y)$ such that

$$
\langle T x-T y, j(x-y)\rangle \leq\|x-y\|^{2} .
$$

The mapping $T$ is pseudocontractive if and only if $(I-T)$ is accretive and is generalized $\Phi$-pseudocontractive if and only if $(I-T)$ is generalized $\Phi$-accretive.

Definition 1.3. A mapping $A: E \rightarrow E$ is said to be Lipschitz if there exists a constant $L>0$ such that

$$
\|A x-A y\| \leq L\|x-y\|, \forall x, y \in E .
$$

A natural generalization of the class of Lipschitz mappings is that of a generalized Lipschitz map. 
A mapping $A: E \rightarrow E$ is called a generalized Lipschitz map if there exits a constant $L>0$ such that

$$
\|A x-A y\| \leq L(1+\|x-y\|) \forall x, y \in E .
$$

Clearly every Lipschitz map is a generalized Lipschitz map (see e.g. 4]).

Definition 1.4. Let $E$ be a normed space with $\operatorname{dim} E \geq 2$. The modulus of smoothness of $E$ is the function $\rho_{E}:[0, \infty) \rightarrow[0, \infty)$ defined by

$$
\rho_{E}(\tau):=\sup \left\{\frac{\|x+y\|+\|x-y\|}{2}-1:\|x\|=1 ;\|y\|=\tau\right\} .
$$

The space $E$ is called uniformly smooth if and only if

$$
\lim _{t \rightarrow 0} \frac{\rho_{E}(t)}{t}=0
$$

We shall need the following result.

Lemma 1.5. ([9]) Let $E$ be a real Banach space, then for all $x, y \in E$, there exists $j(x+y) \in$ $J(x+y)$ such that

$$
\|x+y\|^{2} \leq\|x\|^{2}+2\langle y, j(x+y)\rangle
$$

\section{Main Results}

Theorem 2.1. Let $E$ be a uniformly smooth real Banach space and let $\left\{A_{i}\right\}_{i=1}^{m}: E \rightarrow E$ be m-generalized Lipschitz mappings with $\mathbb{N}:=\bigcap_{i=1}^{m} N\left(A_{i}\right)=\bigcap_{i=1}^{m}\left\{x \in E: A_{i} x=0\right\} \neq \phi$. Let $\left\{a_{n}\right\}_{n=1}^{\infty},\left\{b_{n}\right\}_{n=1}^{\infty}$ and $\left\{c_{n}\right\}_{n=1}^{\infty}$ be real sequences in $[0,1]$ satisfying $(i) a_{n}+b_{n}+c_{n}=$ $1,($ ii $) \sum_{n=1}^{\infty}\left(b_{n}+c_{n}\right)=\infty,($ iii $) \sum_{n=1}^{\infty} c_{n}<\infty,($ iv $) \lim _{n \rightarrow \infty}\left(b_{n}+c_{n}\right)=0,(v) \lim _{n \rightarrow \infty} c_{n}=$ 0 and $\alpha_{n} L<1$. Let $A_{1}$ be generalized $\Phi$-accretive operator and $\left\{x_{n}\right\}_{n=0}^{\infty}$ be the sequence generated for $x_{0} \in E$ by

$$
\begin{aligned}
x_{n+1} & =a_{n} x_{n}+b_{n} S_{1} y_{1 n}+c_{n} u_{n}, \\
y_{1 n} & =a_{n} x_{n}+b_{n} S_{2} y_{2 n} \\
\vdots & \\
y_{(m-2) n} & =a_{n} x_{n}+b_{n} S_{m-1} y_{(m-1) n} \\
y_{(m-1) n} & =a_{n} x_{n}+b_{n} S_{m} x_{n}, \quad m \geq 2, n \geq 1,
\end{aligned}
$$

where $S_{i}: E \rightarrow E, i=1, \cdots, m$ is defined by $S_{i} x:=x-A_{i} x, \forall x \in E$ and $\left\{u_{n}\right\}$ is an arbitrary bounded sequence in $E$. Then, there exists $\gamma_{0} \in R$ such that if $b_{n}+c_{n}<\gamma_{0}, \forall n \geq 0$, the sequence $\left\{x_{n}\right\}$ converges strongly to a common zero of the finite family $\left\{A_{i}\right\}_{i=1}^{m}$.

Proof. Let $x^{*} \in \mathbb{N}:=\bigcap_{n=1}^{m} N\left(A_{i}\right)$. The uniqueness follows from the definition 1.1 . We observe here that $A_{i} x^{*}=0$ if and only if $S_{i} x^{*}=x^{*}(i=1,2 \cdots m)$.

Since $A_{1}$ is a generalized Lipschitz, generalized $\Phi$-accretive mapping, so there exists a strictly increasing function $\Phi:[0, \infty) \rightarrow[0, \infty), \Phi(0)=0$ and $j\left(x-x^{*}\right) \in J\left(x-x^{*}\right)$ such that

$$
\left\|S_{1} x_{n}-S_{1} x^{*}\right\| \leq L\left(1+\left\|x_{n}-x^{*}\right\|\right)
$$

and

$$
\left\langle S_{1} x_{n}-S_{1} x^{*}, j\left(x_{n}-x^{*}\right)\right\rangle \leq\left\|x_{n}-x^{*}\right\|^{2}-\Phi\left(\left\|x_{n}-x^{*}\right\|\right)
$$


Let $\alpha_{n}:=b_{n}+c_{n}$, so that (2.1) reduces to,

$$
\begin{aligned}
x_{n+1} & =\left(1-\alpha_{n}\right) x_{n}+\alpha_{n} S_{1} y_{1 n}+c_{n}\left(u_{n}-S_{1} y_{1 n},\right. \\
y_{1 n} & =\left(1-\alpha_{n}\right) x_{n}+\alpha_{n} S_{2} y_{2 n} \\
\vdots & \\
y_{(m-2) n} & =\left(1-\alpha_{n}\right) x_{n}+\alpha_{n} S_{m-1} y_{(m-1) n} \\
y_{(m-1) n} & =\left(1-\alpha_{n}\right) x_{n}+\alpha_{n} S_{m} x_{n}, \quad m \geq 2, n \geq 1 .
\end{aligned}
$$

We first show that $\left\{x_{n}\right\}_{n=1}^{\infty}$ is bounded.

For this, if $x_{n_{0}}=S_{1} x_{n_{0}}, \forall n \geq 1$, then it clearly holds. So, let if possible, there exists a positive integer $n_{0}$ such that $x_{n_{0}} \neq S_{1} x_{n_{0}}$, thus set $x_{n_{0}}=x_{0}$ and $a_{0}:=\left\|x_{0}-S_{1} x_{0}\right\|\left\|x_{0}-x^{*}\right\|$. Thus by $(2.2)$,

$$
\left\langle S_{1} x_{0}-S_{1} x^{*}, j\left(x_{0}-x^{*}\right)\right\rangle \leq\left\|x_{0}-x^{*}\right\|^{2}-\Phi\left(\left\|x_{0}-x^{*}\right\|\right)
$$

so that, on simplifying

$$
\left\|x_{0}-x^{*}\right\| \leq \Phi^{-1}\left(a_{0}\right)
$$

Since $j$ is uniformly continuous on bounded subsets of $E$, given

$$
\epsilon_{0}:=\frac{\Phi\left(\frac{1}{2} \Phi^{-1}\left(a_{0}\right)\right)}{4\left[2(1+L M) \Phi^{-1}(a 0)+L(1+M)\right]}>0, \exists \delta>0,
$$

such that $\|x-y\|<\delta \Rightarrow\|j(x)-j(y)\|<\epsilon, \forall x, y \in B_{R}(0)$, for some $R>0$. Let $N^{*}:=$ $\sup _{n}\left\|u_{n}-x^{*}\right\|$ and define

$\gamma_{0}:=\min \frac{1}{2}\left\{1, \frac{\Phi^{-1}\left(a_{0}\right)}{3\left[2(1+2 L) \Phi^{-1}\left(a_{0}\right)+2 L\right]}, \frac{\Phi^{-1}\left(a_{0}\right)}{3 N^{*}}, \frac{\delta}{2 \Phi^{-1}\left(a_{0}\right)(2+3 L M)+M+2 L(1+M)+N^{*}}\right\}$.

Now, we claim that $\left\|x_{n}-x^{*}\right\| \leq 2 \Phi^{-1}\left(a_{0}\right), \forall n \geq 0$. Clearly in view of (2.4), the claim holds for $n=0$.

We next assume that $\left\|x_{n}-x^{*}\right\| \leq 2 \Phi^{-1}\left(a_{0}\right)$, for some $n$ and we shall prove that $\left\|x_{n+1}-x^{*}\right\| \leq$ $2 \Phi^{-1}\left(a_{0}\right)$.

Let if possible, this is not true, i.e. $\left\|x_{n+1}-x^{*}\right\|>2 \Phi^{-1}\left(a_{0}\right)$. 
Thus we have,

$$
\begin{aligned}
\left\|y_{1 n}-x^{*}\right\|= & \left\|\left(1-\alpha_{n}\right)\left(x_{n}-x^{*}\right)+\alpha_{n}\left(S_{2} y_{2 n}-x^{*}\right)\right\| \\
\leq & \left(1-\alpha_{n}\right)\left\|x_{n}-x^{*}\right\|+\alpha_{n} L\left(1+\left\|y_{2 n}-x^{*}\right\|\right) \\
\leq & \left(1-\alpha_{n}\right)\left\|x_{n}-x^{*}\right\|+\alpha_{n} L+\alpha_{n} L\left[\left(1-\alpha_{n}\right)\left\|x_{n}-x^{*}\right\|+\alpha_{n}\left\|S_{3} y_{3 n}-x^{*}\right\|\right] \\
\leq & \left(1-\alpha_{n}\right)\left\|x_{n}-x^{*}\right\|+\alpha_{n} L+\alpha_{n} L\left[\left(1-\alpha_{n}\right)\left\|x_{n}-x^{*}\right\|+\alpha_{n} L\left(1+\left\|y_{3 n}-x^{*}\right\|\right)\right] \\
\leq & \left\|x_{n}-x^{*}\right\|+\alpha_{n} L+\alpha_{n} L\left[\left\|x_{n}-x^{*}\right\|+\alpha_{n} L\left(1+\left\|y_{3 n}-x^{*}\right\|\right)\right] \\
= & \left\|x_{n}-x^{*}\right\|+\alpha_{n} L\left\|x_{n}-x^{*}\right\|+\alpha_{n}^{2} L^{2}\left\|y_{3 n}-x^{*}\right\|+\alpha_{n} L+\alpha_{n}^{2} L^{2} \\
\vdots & \\
\leq & \left(1+\alpha_{n} L+\alpha_{n}^{2} L^{2}+\cdots+\alpha_{n}^{m-1} L^{m-1}\right)\left\|x_{n}-x^{*}\right\| \\
& +\left(\alpha_{n} L+\alpha_{n}^{2} L^{2}+\cdots+\alpha_{n}^{m-1} L^{m-1}\right) \\
\leq & \left(1+\alpha_{n} L+\alpha_{n}^{2} L^{2}+\cdots+\alpha_{n}^{m-1} L^{m-1}\right)\left\|x_{n}-x^{*}\right\| \\
& +\left(1+\alpha_{n} L+\alpha_{n}^{2} L^{2}+\cdots+\alpha_{n}^{m-1} L^{m-1}\right) \\
\leq & \frac{\left(1-\alpha_{n}^{m} L^{m}\right)}{1-\alpha_{n} L}\left\|x_{n}-x^{*}\right\|+\frac{\left(1-\alpha_{n}^{m} L^{m}\right)}{1-\alpha_{n} L} \\
\leq & M\left\|x_{n}-x^{*}\right\|+M
\end{aligned}
$$

Thus since, $\left\|x_{n}-x^{*}\right\| \leq 2 \Phi^{-1}\left(a_{0}\right)$, so

$$
\left\|y_{1 n}-x^{*}\right\| \leq M\left(1+2 \Phi^{-1}\left(a_{0}\right)\right)
$$

Also we have the following relations,

(i)

$$
\begin{aligned}
\left\|x_{n}-S_{1} y_{1 n}\right\| & \leq\left\|x_{n}-x^{*}\right\|+\left\|S_{1} y_{1 n}-x^{*}\right\| \\
& \leq\left\|x_{n}-x^{*}\right\|+L\left(1+\left\|y_{1 n}-x^{*}\right\|\right) \\
& \leq\left\|x_{n}-x^{*}\right\|+L\left(1+M\left\|x_{n}-x^{*}\right\|+M\right) \\
& \leq L(1+M)+2(1+L M) \Phi^{-1}\left(a_{0}\right),
\end{aligned}
$$

(ii) $\left\|y_{1 n}-x^{*}\right\| \geq \frac{1}{2} \Phi^{-1}\left(a_{0}\right)$

(iii) $\left\|x_{n+1}-x^{*}\right\| \leq 3 \Phi^{-1}\left(a_{0}\right)$

(iv) $\left\|x_{n}-x^{*}\right\| \geq \Phi^{-1}\left(a_{0}\right)$ 
$(\mathrm{v})$

$$
\begin{aligned}
\left\|U_{n}\right\|\left\|y_{1 n}-x^{*}\right\| & =\left\|c_{n}\left(u_{n}-S_{1} y_{1 n}\right)\right\|\left\|y_{1 n}-x^{*}\right\| \\
& \leq c_{n}\left\|y_{1 n}-x^{*}\right\|\left[\left\|u_{n}-x^{*}\right\|+\left\|S_{1} y_{1 n}-x^{*}\right\|\right] \\
& \leq c_{n}\left\|y_{1 n}-x^{*}\right\|\left[N^{*}+L\left(1+\left\|y_{1 n}-x^{*}\right\|\right)\right] \\
& \leq c_{n} M\left(1+2 \Phi^{-1}\left(a_{0}\right)\right)\left[N^{*}+L\left(1+M\left(1+2 \Phi^{-1}\left(a_{0}\right)\right)\right)\right] \\
& \leq c_{n} M_{1}
\end{aligned}
$$

Thus from the recursion formula (2.3) and using the above relations, we have

$$
\begin{aligned}
\left\|x_{n+1}-x^{*}\right\|^{2}= & \left\|x_{n}-x^{*}-\left[\alpha_{n}\left(x_{n}-S_{1} y_{1 n}\right)-U_{n}\right]\right\|^{2} \\
\leq & \left\|x_{n}-x^{*}\right\|^{2}-2 \alpha_{n}\left\langle x_{n}-S_{1} y_{1 n}, j\left(x_{n+1}-x^{*}\right)\right\rangle+2\left\langle U_{n}, j\left(x_{n+1}-x^{*}\right)\right\rangle \\
\leq & \left\|x_{n}-x^{*}\right\|^{2}-2 \alpha_{n}\left\langle x_{n}-S_{1} y_{1 n}, j\left(x_{n+1}-x^{*}\right)-j\left(y_{1 n}-x^{*}\right)\right\rangle \\
& -2 \alpha_{n}\left\langle x_{n}-S_{1} y_{1 n}, j\left(y_{1 n}-x^{*}\right)\right\rangle+2\left\|U_{n}\right\|\left\|j\left(x_{n+1}-x^{*}\right)-j\left(y_{1 n}-x^{*}\right)\right\| \\
& +2\left\|U_{n}\right\|\left\|y_{1 n}-x^{*}\right\| \\
\leq & \left\|x_{n}-x^{*}\right\|^{2}+2 \alpha_{n}\left\|x_{n}-S_{1} y_{1 n}\right\|\left\|j\left(x_{n+1}-x^{*}\right)-j\left(y_{1 n}-x^{*}\right)\right\| \\
& +2 \alpha_{n}\left\|x_{n}-y_{1 n}\right\|\left\|y_{1 n}-x^{*}\right\|-2 \alpha_{n} \Phi\left(\left\|y_{1 n}-x^{*}\right\|\right) \\
& +2\left\|U_{n}\right\|\left\|j\left(x_{n+1}-x^{*}\right)-j\left(y_{1 n}-x^{*}\right)\right\|+2\left\|U_{n}\right\|\left\|y_{1 n}-x^{*}\right\| \\
\leq & \left\|x_{n}-x^{*}\right\|^{2}-2 \alpha_{n} \Phi\left(\frac{1}{2} \Phi^{-1}\left(a_{0}\right)\right)+2 M c_{n} \\
& +2 \alpha_{n}\left[L(1+M)+2\left(1+L M^{-1}\left(a_{0}\right)\right]\left\|j\left(x_{n+1}-x^{*}\right)-j\left(y_{1 n}-x^{*}\right)\right\|\right. \\
& +2 c_{n}\left[N^{*}+L\left(1+M\left(1+2 \Phi^{-1}\left(a_{0}\right)\right)\right]\left\|j\left(x_{n+1}-x^{*}\right)-j\left(y_{1 n}-x^{*}\right)\right\|\right. \\
& +2 \alpha_{n} M\left(1+2 \Phi^{-1}\left(a_{0}\right)\right)\left[2 \Phi^{-1}\left(a_{0}\right)+M\left(1+2 \Phi^{-1}\left(a_{0}\right)\right)\right]
\end{aligned}
$$

Set $M_{2}:=2 M_{1}+2\left[N^{*}+L\left(1+M\left(1+2 \Phi^{-1}\left(a_{0}\right)\right)\right] \epsilon_{0}\right.$ and $M_{3}:=2 M\left(1+2 \Phi^{-1}\left(a_{0}\right)\right)\left[2 \Phi^{-1}\left(a_{0}\right)+M\left(1+2 \Phi^{-1}\left(a_{0}\right)\right)\right]$ such that $M_{2}+M_{3}<\frac{1}{2} \Phi\left(\frac{1}{2} \Phi^{-1}\left(a_{0}\right)\right)$.

Here we observe that, since $\left(x_{n+1}-x^{*}\right),\left(y_{1 n}-x^{*}\right) \in B_{R}(0)$, for $R:=3 \Phi^{-1}\left(a_{0}\right)>0$ and also

$$
\begin{aligned}
\left\|x_{n+1}-y_{1 n}\right\| \leq & \left\|x_{n}-y_{1 n}\right\|+\alpha_{n}\left\|x_{n}-S_{1} y_{1 n}\right\|+c_{n}\left\|u_{n}-x^{*}\right\|+c_{n}\left\|S_{1} y_{1 n}-x^{*}\right\| \\
\leq & \alpha_{n}\left\|x_{n}-S_{2} y_{2 n}\right\|+\alpha_{n}\left\|x_{n}-S_{1} y_{1 n}\right\|+c_{n}\left\|u_{n}-x^{*}\right\|+c_{n} L\left(1+\left\|y_{1 n}-x^{*}\right\|\right) \\
\leq & \alpha_{n}\left[\left\|x_{n}-x^{*}\right\|+\left\|S_{2} y_{2 n}-x^{*}\right\|\right]+\alpha_{n}\left\|x_{n}-S_{1} y_{1 n}\right\|+c_{n}\left\|u_{n}-x^{*}\right\| \\
& +c_{n} L\left(1+\left\|y_{1 n}-x^{*}\right\|\right) \\
\leq & \alpha_{n}\left[2 \Phi^{-1}\left(a_{0}\right)+M\left(1+2 L \Phi^{-1} a_{0}\right)\right] \\
& +\alpha_{n}\left[L(1+M)+2(1+L M) \Phi^{-1}\left(a_{0}\right)\right]+c_{n} N^{*}+c_{n} L\left(1+M\left(1+2 \Phi^{-1}\left(a_{0}\right)\right)\right) \\
\leq & \gamma_{0}\left[2 \Phi^{-1}\left(a_{0}\right)(2+3 L M)+M+2 L(1+M)+N^{*}\right] \\
< & \delta
\end{aligned}
$$

so that,

$$
\left\|j\left(x_{n+1}-x^{*}\right)-j\left(y_{1 n}-x^{*}\right)\right\|<\epsilon_{0} .
$$


Hence from $(2.7)$,

$$
\begin{aligned}
\left\|x_{n+1}-x^{*}\right\|^{2} \leq & \left\|x_{n}-x^{*}\right\|^{2}-2 \alpha_{n} \Phi\left(\frac{1}{2} \Phi^{-1}\left(a_{0}\right)\right)+M_{2} c_{n}+\alpha_{n} M_{3} \\
& +2 \alpha_{n}\left[L(1+M)+2(1+L M) \Phi^{-1}\left(a_{0}\right)\right] \epsilon_{0} \\
\leq & \left\|x_{n}-x^{*}\right\|^{2}-2 \alpha_{n} \Phi\left(\frac{\Phi^{-1}\left(a_{0}\right)}{2}\right)+\alpha_{n}\left(M_{3}+M_{2}\right)+\frac{1}{2} \alpha_{n} \Phi\left(\frac{\Phi^{-1}\left(a_{0}\right)}{2}\right) \\
\leq & \left\|x_{n}-x^{*}\right\|^{2}-2 \alpha_{n} \Phi\left(\frac{\Phi^{-1}\left(a_{0}\right)}{2}\right)+\alpha_{n} \Phi\left(\frac{\Phi^{-1}\left(a_{0}\right)}{2}\right) \\
\leq & \left\|x_{n}-x^{*}\right\|^{2}-\alpha_{n} \Phi\left(\frac{\Phi^{-1}\left(a_{0}\right)}{2}\right) \\
\leq & \left\|x_{n}-x^{*}\right\|^{2} \\
\leq & \left(2 \Phi^{-1}\left(a_{0}\right)\right)^{2}
\end{aligned}
$$

which is a contradiction.

Hence $\left\{x_{n}\right\}_{n=1}^{\infty}$ and thus $\left\{y_{i n}\right\}_{n=1}^{\infty}$ is bounded. Since $S_{1}$ is a bounded operator, so $\left\{S_{1} x_{n}\right\}$ and $\left\{S_{1} y_{i n}\right\}$ is also bounded.

Let $M:=\sup \left\{2\left\|x_{n}-y_{i n}\right\|\left\|y_{i n}-x^{*}\right\|\right\}, \rho:=\sup \left\|x_{n}-S_{1} y_{i n}\right\|, \beta:=\sup \left\|u_{n}-S_{1} y_{i n}\right\|$ and $\sigma:=\sup \left\{2\left\|u_{n}-S_{1} x_{n}\right\|\left\|j\left(x_{n+1}-x^{*}\right)-j\left(y_{1 n}-x^{*}\right)\right\|+2\left\|u_{n}-S_{1} x_{n}\right\|\left\|y_{1 n}-x^{*}\right\|\right\}$,

so that from $(2.3)$,

$$
\begin{aligned}
\left\|x_{n+1}-x^{*}\right\|^{2} \leq & \left\|x_{n}-x^{*}\right\|^{2}-2 \alpha_{n} \Phi\left(\left\|y_{1 n}-x^{*}\right\|\right)+2 \alpha_{n}\left\|x_{n}-y_{1 n}\right\|\left\|y_{1 n}-x^{*}\right\| \\
& +2 \alpha_{n}\left\|x_{n}-S_{1} y_{1 n}\right\|\left\|j\left(x_{n+1}-x^{*}\right)-j\left(y_{1 n}-x^{*}\right)\right\| \\
& +2\left\|U_{n}\right\|\left\|j\left(x_{n+1}-x^{*}\right)-j\left(y_{1 n}-x^{*}\right)\right\|+2\left\|U_{n}\right\|\left\|y_{1 n}-x^{*}\right\| \\
\leq & \left\|x_{n}-x^{*}\right\|^{2}-2 \alpha_{n} \Phi\left(\left\|y_{1 n}-x^{*}\right\|\right)+c_{n} \sigma+\alpha_{n} M \\
& +2 \alpha_{n} \rho\left\|j\left(x_{n+1}-x^{*}\right)-j\left(y_{1 n}-x^{*}\right)\right\|
\end{aligned}
$$

Next here we claim that $\inf \left\{\left\|y_{1 n}-x^{*}\right\|, n \geq 0\right\}=0$.

For this, let if possible, $\liminf \operatorname{in}_{n \rightarrow \infty}\left\|y_{1 n}-x^{*}\right\|=2 \delta>0$.

Then there exists a positive integer $N_{1}$ such that

$$
\left\|y_{1 n}-x^{*}\right\| \geq \delta, \forall n \geq N_{1} \text { i.e. } \Phi\left(\left\|y_{1 n}-x^{*}\right\|\right) \geq \Phi(\delta), \forall n \geq N_{1} .
$$

Again since,

$$
\begin{aligned}
\left\|x_{n+1}-y_{1 n}\right\| & =\left\|\left(1-\alpha_{n}\right) x_{n}-y_{1 n}+\alpha_{n} S_{1} y_{1 n}+c_{n}\left(u_{n}-S_{1} y_{1 n}\right)\right\| \\
& \leq\left\|x_{n}-y_{1 n}\right\|+\alpha_{n}\left\|x_{n}-S_{1} y_{1 n}\right\|+c_{n}\left\|u_{n}-S_{1} y_{1 n}\right\| \\
& \leq \alpha_{n}\left\|x_{n}-S_{2} y_{2 n}\right\|+\alpha_{n} \rho+c_{n} \beta \\
& \leq 2 \alpha_{n} \rho+c_{n} \beta \\
& \rightarrow 0 \text { as } n \rightarrow \infty,
\end{aligned}
$$

and $j$ is uniformly continuous on bounded subsets, so $\left\|j\left(x_{n+1}-x^{*}\right)-j\left(y_{1 n}-x^{*}\right)\right\|<\frac{\Phi(\delta)}{2 \rho}, \forall n \geq N_{1}$. 
Hence for all $n \geq N_{1}$, we have,

$$
\begin{aligned}
\left\|x_{n+1}-x^{*}\right\|^{2} & \leq\left\|x_{n}-x^{*}\right\|^{2}-2 \alpha_{n} \Phi(\delta)+2 \alpha_{n} \rho \frac{\Phi(\delta)}{2 \rho}+c_{n} \sigma+\alpha_{n} M \\
& =\left\|x_{n}-x^{*}\right\|^{2}-\alpha_{n} \Phi(\delta)+c_{n} \sigma+\alpha_{n} M
\end{aligned}
$$

thus,

$$
(\Phi(\delta)-M) \sum_{j=1}^{n} \alpha_{j} \leq \sum_{j=1}^{n}\left(\left\|x_{n}-x^{*}\right\|^{2}-\left\|x_{n+1}-x^{*}\right\|^{2}\right)+\sigma \sum_{j=1}^{n} c_{n}
$$

But since $\sum_{j=1}^{\infty} c_{j}<\infty$, this implies that $\sum_{j=1}^{\infty} \alpha_{j}<\infty$, a contradiction.

Thus our claim is true.

So there exists a subsequence $\left\{y_{1 n_{j}}-x^{*}\right\}$ of $\left\{y_{1 n}-x^{*}\right\}$ such that $\lim _{j \rightarrow \infty}\left\|y_{1 n_{j}}-x^{*}\right\|=0$ and thus from $(2.3), \lim _{j \rightarrow \infty}\left\|x_{n_{j}}-x^{*}\right\|=0$. Hence for given $\epsilon_{0}>0$, there exists a positive integer $n_{j_{0}}>N_{1}$ such that $\left\|x_{n_{j}}-x^{*}\right\|<\epsilon, \frac{\alpha_{n_{j}}\left(1-2 \alpha_{n_{j}} L\right) W+\alpha_{n_{j}} L}{1-\alpha_{n_{j}} L}<\frac{\epsilon}{4}, \forall n_{j}>n_{j_{0}}$, where $W:=\sup \left\{\left\|x_{n}-x^{*}\right\|\right\}$. Again choose a positive integer $n_{0}>n_{j_{0}}$ such that $\rho \alpha_{n}<\frac{\epsilon}{8}, c_{n}=$ $\min \left\{\frac{\epsilon}{4 \beta}, \frac{\alpha_{n}}{4 \sigma} \Phi\left(\frac{\epsilon}{2}\right)\right\}, M<\frac{1}{4 \sigma} \Phi\left(\frac{\epsilon}{2}\right)$ and $\left\|j\left(x_{n_{j}+1}-x^{*}\right)-j\left(x_{n_{j}}-x^{*}\right)\right\|<\frac{1}{2 \rho} \Phi\left(\frac{\epsilon}{2}\right)$. We next claim that

$$
\left\|x_{n_{j}+m}-x^{*}\right\|<\epsilon, n_{j}>n_{j_{0}}, \forall m \geq 1
$$

We prove it by induction. For this, we first prove that $\left\|x_{n_{j}+1}-x^{*}\right\|<\epsilon$.

If not, then $\exists n_{j_{1}}>n_{j_{0}}$ such that $\left\|x_{n_{j}+1}-x^{*}\right\| \geq \epsilon$.

Thus,

$$
\begin{aligned}
\left\|x_{n_{j}}-x^{*}\right\| & \geq\left\|x_{n_{j}+1}-x^{*}\right\|-\alpha_{n_{j}}\left\|x_{n_{j}}-S_{1} y_{1 n_{j}}\right\|-c_{n_{j}}\left\|u_{n}-S_{1} y_{1 n_{j}}\right\| \\
& \geq \epsilon-\alpha_{n_{j}} \rho-c_{n_{j}} \beta \\
& >\frac{3 \epsilon}{4}
\end{aligned}
$$

Also,

$$
\begin{aligned}
\left\|y_{1 n_{j}}-x^{*}\right\| & \geq\left(1-\alpha_{n}\right)\left(\frac{1-2 \alpha_{n} L}{1-\alpha_{n} L}\right)\left\|x_{n_{j}}-x^{*}\right\|-\left(\frac{\alpha_{n} L}{1-\alpha_{n} L}\right) \\
& =\left\|x_{n_{j}}-x^{*}\right\|-\alpha_{n} \frac{\left(1-2 \alpha_{n} L\right)}{1-\alpha_{n} L}\left\|x_{n_{j}}-x^{*}\right\|-\frac{\alpha_{n} L}{1-\alpha_{n} L} \\
& >\frac{3 \epsilon}{4}-\frac{\alpha_{n_{j}}\left(1-2 \alpha_{n_{j}} L\right) W+\alpha_{n_{j}} L}{1-\alpha_{n_{j}} L} \\
& >\frac{\epsilon}{2}
\end{aligned}
$$

which implies,

$$
\Phi\left(\left\|y_{1 n_{j}}-x^{*}\right\|\right) \geq \Phi\left(\frac{\epsilon}{2}\right), \forall n_{j}>n_{j_{0}} .
$$


Thus using the recursion formula (2.3), we have

$$
\begin{aligned}
\left\|x_{n_{j}+1}-x^{*}\right\|^{2} & \leq\left\|x_{n_{j}}-x^{*}\right\|^{2}-2 \alpha_{n_{j}} \Phi\left(\left\|y_{1 n_{j}}-x^{*}\right\|\right)+c_{n_{j}} \sigma+\alpha_{n_{j}} M \\
& +2 \rho \alpha_{n_{j}}\left\|j\left(x_{n_{j}+1}-x^{*}\right)-j\left(y_{n_{n_{j}}}-x^{*}\right)\right\| \\
& \leq\left\|x_{n_{j}}-x^{*}\right\|^{2}-2 \alpha_{n_{j}} \Phi\left(\frac{\epsilon}{2}\right)+c_{n_{j}} \sigma+\alpha_{n_{j}} M+2 \rho \alpha_{n_{j}} \frac{\Phi\left(\frac{\epsilon}{2}\right)}{2 \rho} \\
& <\epsilon^{2}-2 \alpha_{n_{j}} \Phi\left(\frac{\epsilon}{2}\right)+\frac{\alpha_{n_{j}} \Phi\left(\frac{\epsilon}{2}\right)}{2}+\alpha_{n_{j}} \Phi\left(\frac{\epsilon}{2}\right) \\
& =\epsilon^{2}-\frac{\alpha_{n_{j}} \Phi\left(\frac{\epsilon}{2}\right)}{2} \\
& <\epsilon^{2}
\end{aligned}
$$

which is a contradiction.

Hence the claim is true for $m=1$. Let us assume that it holds for some $m=k$. Then following the above steps, we can easily show that it holds for $m=k+1$. Hence the claim holds for all $m \geq 1$. This implies that the sequence $\left\{x_{n}\right\}$ converges strongly to the common zero $x^{*}$ as $n \rightarrow \infty$.

Remark 2.2. If the family $\left\{A_{i}\right\}_{i=1}^{m}$ of generalized Lipschitz map be such that $A_{1}=A_{2}=$ $\cdots=A_{m}=A$, where $A$ is generalized $\Phi$-accretive, then the result of [4] holds as a special case of our theorem.

Theorem 2.3. Let $E$ be a uniformly smooth real Banach space and let $\left\{T_{i}\right\}_{i=1}^{m}: E \rightarrow E$ be m-generalized Lipschitz mappings with $F:=\bigcap_{i=1}^{m} F\left(T_{i}\right)=\bigcap_{i=1}^{m}\left\{x \in E: T_{i} x=x\right\} \neq \phi$. Let $\left\{a_{n}\right\}_{n=1}^{\infty},\left\{b_{n}\right\}_{n=1}^{\infty}$ and $\left\{c_{n}\right\}_{n=1}^{\infty}$ be real sequences in $[0,1]$ satisfying $(i) a_{n}+b_{n}+c_{n}=$ $1,($ ii $) \sum_{n=1}^{\infty}\left(b_{n}+c_{n}\right)=\infty,($ iii $) \sum_{n=1}^{\infty} c_{n}<\infty,($ iv $) \lim _{n \rightarrow \infty}\left(b_{n}+c_{n}\right)=0,(v) \lim _{n \rightarrow \infty} c_{n}=$ 0 and $\alpha_{n} L<1$. Let $T_{1}$ be generalized $\Phi$-accretive operator and $\left\{x_{n}\right\}_{n=0}^{\infty}$ be the sequence generated for $x_{0} \in E$, by

$$
\begin{aligned}
x_{n+1} & =a_{n} x_{n}+b_{n} T_{1} y_{1 n}+c_{n} u_{n}, \\
y_{1 n} & =a_{n} x_{n}+b_{n} T_{2} y_{2 n} \\
\vdots & \\
y_{(m-2) n} & =a_{n} x_{n}+b_{n} T_{m-1} y_{(m-1) n} \\
y_{(m-1) n} & =a_{n} x_{n}+b_{n} T_{m} x_{n}, \quad m \geq 2, n \geq 1,
\end{aligned}
$$

where $\left\{u_{n}\right\}$ is an arbitrary bounded sequence in $E$. Then, the sequence $\left\{x_{n}\right\}$ converges strongly to a common fixed point of the finite family $\left\{T_{i}\right\}_{i=1}^{m}$.

Proof. Since we know that a mapping $T$ is generalized $\Phi$-pseudocontractive if and only if $(I-T)$ is $\Phi$-accretive. Thus the proof follows from Theorem 2.1, replacing $S_{i}$ by $T_{i}$.

\section{ACKNOWLEDGMENTS}

This research is supported under UGC(JRF) scheme, India. 


\section{REFERENCES}

[1] F.E. Browder, Nonlinear mappings of nonexpansive and accretive type in Banach space, Bull. Amer. Math. Soc., 73 (1967), 875-882.

[2] F.E. Browder and W.V. Petryshyn, Construction of fixed points of nonlinear mappings in Hilbert space, J. Math. Anal. Appl., 20 (1967), 197-228.

[3] S.S. Chang, K.K. Tan, H.W.J. Lee and C.K. Chan, On the convergnce of implicit iteration process wih error for a finite family of asymptotically nonexpansive mappings, J. Math. Anal. Appl., 313 (2006), $273-283$.

[4] C.E. Chidume and C.O. Chidume, Convergence theorems for zeros of generalized Lipschitz generalized $\Phi$-quasi accretive operators, Proc. Amer. Math. Soc., 134 (2006), 243-251.

[5] H. Hirano and Z. Huang, Convergence theorems for multi-valued $\phi$-hemicontractive operators and $\phi$ strongly accretive operators, Comp. Math. Appl., 46 (2003), 1461-1471.

[6] J.S. Jung, Iterative approaches to common fixed points of nonexpansive mappings in Banach spaces, J. Math. Anal. Appl., 302 (2005), 509-520.

[7] R.De Marr, Common fixed points for commuting contraction mappings, Pacific J. Math., 53 (1974), 487-493.

[8] A. Markov, Quelques theorems sur les ensembles abeliens, Dokl. Akad. Nauk. SSSR (N.S.), 10 (1936), 311-314.

[9] W.V. Petryshyn, A characterization of strict convexity of Banach spaces and other uses of duality mappings, J. Func. Anal., 6 (1970), 282-291.

[10] Z. Sun, Strong convergence of an implicit ieration process for a finite family of asymptotically quasinonexpansive mappings, J. Math. Anal. Appl., 286 (2003), 351-358.

[11] Y. Xu, Ishikawa and Mann iterative processses with erors for nonlinear strongly accretive operator equations, J. Math. Anal. Appl., 224 (1998), 91-101.

[12] L.C. Zhao and S.S. Chang, Strong convergence theorems for equilibrium problems and fixed point problems, J. Nonlinear Sci. Appl., 2 (2009), 78-91.

[13] H. Zhou, L. Wei and Y.J. Cho, Strong convrgence theorems on an iteraive method for family of finite nonexpansive mappings in reflexive Banach spaces, Appl. Math. Comput., 173 (2006), 196-212.

School of Studies in Mathematics, Pt. Ravishankar Shukla University Raipur - 492010 (C.G.), INDIA

E-mail address: ${ }^{1}$ niyati.kuhu@gmail.com, ${ }^{2}$ sharmabk07@gmail.com 